\title{
Underwater ROV as Inspection and Development Platform
}

\section{Marko Vukšića, Slaven Josipovića ${ }^{a}$ Ante Čorićb ${ }^{\text {, Ante Kraljevića }}$}

The successful business technical cooperation between the University of Split and the company PLOVPUT LLC yields a very usable, lightweight, maneuverable underwater Remote Operated Vehicle (ROV). The ROV is capable of diving down to $150 \mathrm{~m}$ depth. It can carry different remote controlled sensors and tools, and resolve challenging tasks. Primarily ROV's usage is to inspect underwater electrical installation. It is equipped with HD camera and LED lights. An umbilical cable is used to transfer data and electrical power from the surface to the underwater vehicle. The position control was realized using inexpensive PS2 joystick console. ROV's development was mostly carried out by the students of the University of Split (UNIST). The mechanical and electrical subsystems were built and tested at UNIST laboratories. In this paper, ROV mechanical and electrical systems are outlined and basic subsystems are presented.

\section{KEY WORDS}

$\sim$ Remote Operated Vehicle (ROV)

$\sim$ Power supply system

$\sim$ Control system

$\sim$ Arduino ATmega.

\author{
a. University of Split, Department of Professional Studies, Split, Croatia \\ e-mail:vuksic.marko@oss.unist.hr \\ b. Plovput d.o.o., Split, Croatia \\ e-mail: antecoric89@gmail.com
}

This work is licensed under (cc) BY

\section{INTRODUCTION}

The Department of Professional Studies, University of Split, (DoPS UNIST), aims at enhancing human resources and increasing their capacity to participate in the development of advanced underwater systems. Along with the researchers, the students are highly encouraged to participate in the application of new and emerging technologies particularly during the final exam and diploma thesis preparation.

The main activities of the company PLOVPUT LLC. are the construction and maintenance of the lighthouses and other navigational equipment in the Adriatic Sea waters of the Republic of Croatia. Because of the need to improve the technology of maintenance and safety when performing these activities, the initiative for the development and prototype design of the remotely operated vehicle (ROV) emerged. Excellent business-technical cooperation of DoPS UNIST and the company PLOVPUT LLC. yields initiative to develop the low-cost ROV capable of fulfilling tasks that stand for company PLOVPUT LLC. The Company took over financing the prototyping, while the mentoring and professional support was offered by DoPS UNIST.

The need for a robotic underwater intervention comes in many forms and it is generally driven by specific tasks (Ferreira et al., 2014; Christ and Wernli, 2007). ROV is often used for the underwater tasks that are more safely and easily carried out without the presence of man, i.e. a diver. The principal objective of the new ROV is to serve as cost-effective inspection and development platform for the field-trials of the novel subsystems for the supervision of underwater electrical installations and cables.

The realized ROV is a result of a joint effort of the students from the Department of Electrical Engineering and students from the Department of Mechanical Engineering at DoPS UNIST.

The main effort of the mechanical construction development, testing and production was taken by the Mechanical Engineering Department students, that of the power 
and control systems development was taken by the Electrical Engineering Department students.

The realized ROV is controlled by the operator from the surface by means of the control unit and hand-held joystick that are connected to sub-sea module through umbilical cable. The PS2 joystick was selected because it is an easily adaptable and very intuitive tool for controlling objects in three axes. The umbilical cable consists of power cable, communication UTP cable and video cable.

ROV belongs to the middle-class submersible remote control vehicles, which is equipped with various sensors with the ability to dive up to a hundred and fifty meters. Because of its modular structural design it leaves room for various extensions and components (sonar, robot manipulator, a system of airworthiness compensation ...). It can be easily reconfigured in agreement with specific tasks.

The realized submersible module is equipped with a camera and a variety of sensors and optional tools. In order to illuminate the underwater environment, two high-luminosity

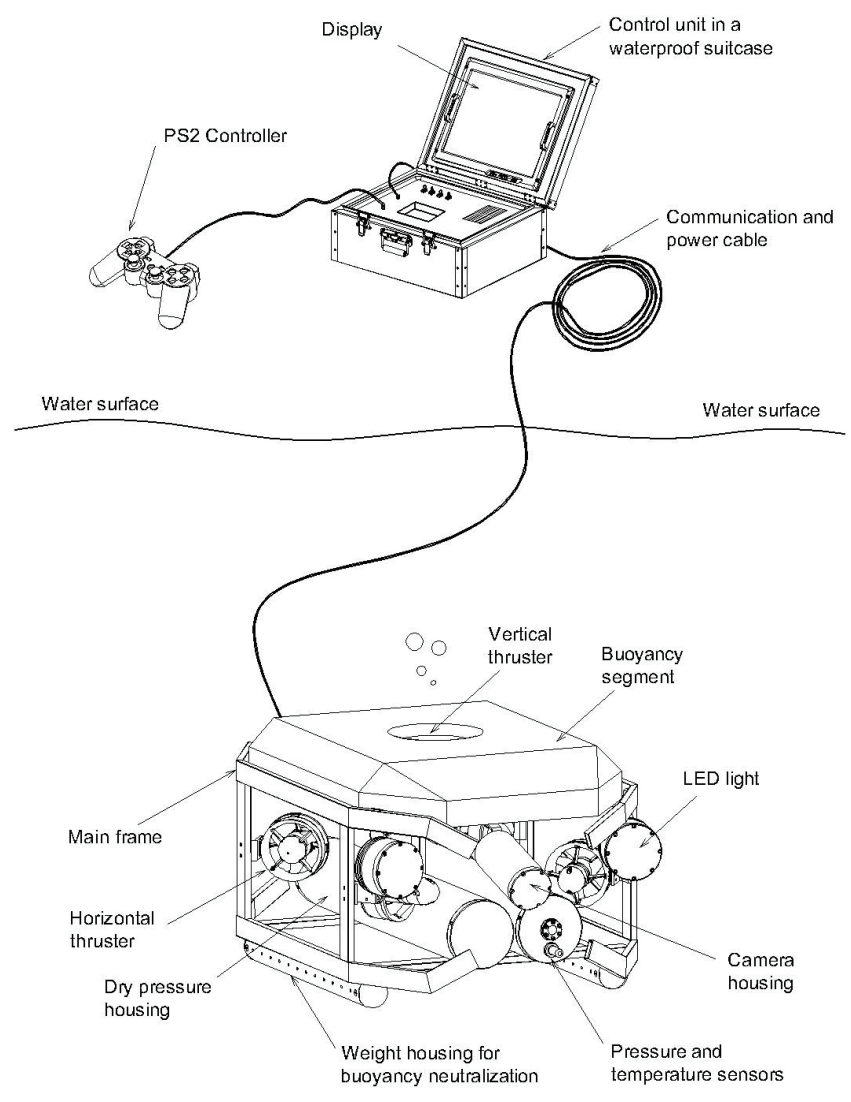

Figure 1.

ROV system. dimmable LED lights were installed to illuminate the space in front of camera.

The ROV is equipped with a propulsion that has six degrees of freedom and ensures a precise position control in all three axes.

The entire system is controlled by the surface computer unit and electronic devices, which collect, organize, and send control information to the sub-sea computer unit via the cable using standard communication protocol,

The basic system requirements of ROV:

- Maximum diving depth: 150 meters;

- Maneuverability, six degrees of freedom;

- Total available power submersibles: 1,000 W;

- Standard equipment: Full HD camera, pressure and depth sensor, temperature sensor, penetration of water into dry housing alarm sensor, compass, accelerometer and gyroscope in three axes, surface video monitor;

- Controllability by handheld PS2 controller.

\section{MECHANICAL DESIGN}

The underwater vehicle, described in this paper, is an observation class ROV, which is designed specifically for lighter usage with propulsion systems, to deliver a camera and sensor package to a place where it can provide a meaningful picture or gather data (Christ and Wernli, 2007).

ROV construction is self-sustained, stainless steel frame with a buoyancy block made of aluminum. The electrical equipment, camera and LED lights were installed into pressure vessels, sealed with non-transparent and transparent lids respectively. The construction is selected as passively stable. The buoyancy block in turn is designed as internally pressure-compensated vessel. The air pressure to the buoyancy block at sea level is 6bar, thus the net pressure to the block body at the depth of $150 \mathrm{~m}$ is 10 bar, reducing the possibility of the block compression.

The system is constructed with a high center of buoyancy and a low center of gravity, to give the camera platform maximum stability about the longitudinal and lateral axis. Roll and pitch movement is minimized by the design. Adjustable lead ballast system located at the bottom of the ROV frame is used to achieve neutral buoyancy, as the opposite uplift force is achieved by the buoyancy block located on the upper side of the ROV (More et al., 2010). The overall vehicle size and the main frame design is a product of optimal space usage around all the important components that include propulsion units, electronic vessels, LED lights, sensors and a camera.

All critical parts of the ROV were subjected to computersimulated stress tests. This was done by the finite element method analysis using CAD software CATIA. All parts were subjected to pressure loads of 16 bar $(150 \mathrm{~m})$ as it is the depth rating (Dobrilo, 2011) defined in the initial requirements list. 


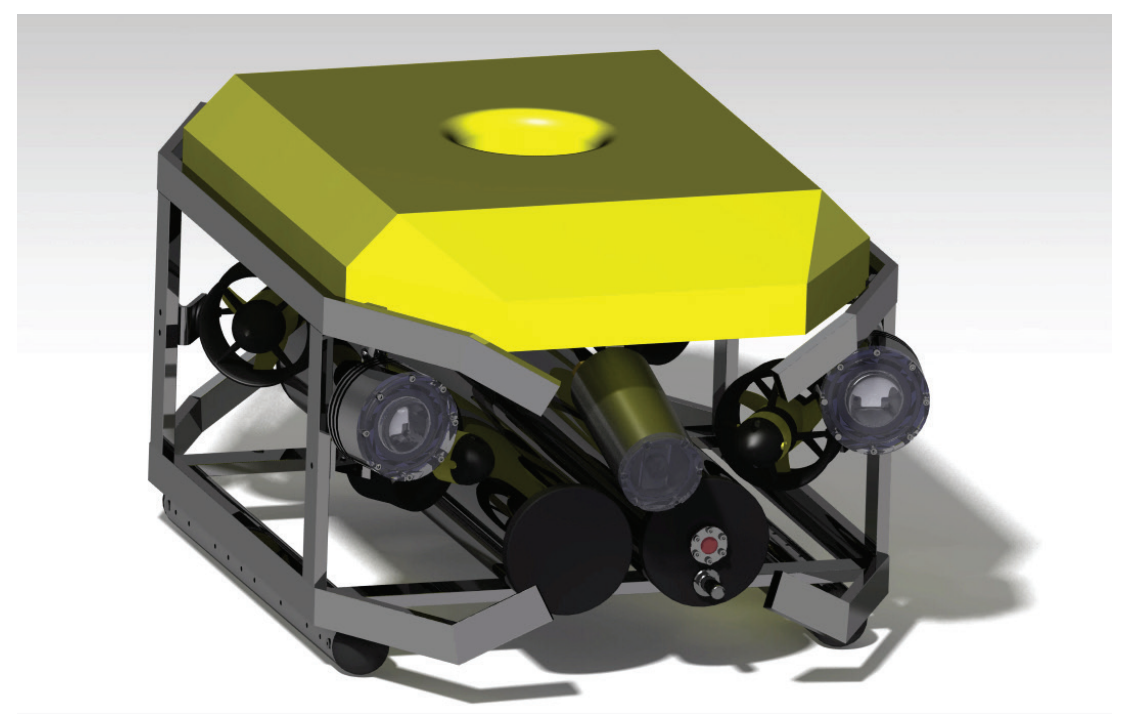

Figure 2.

ROV frame and vessels drawing.

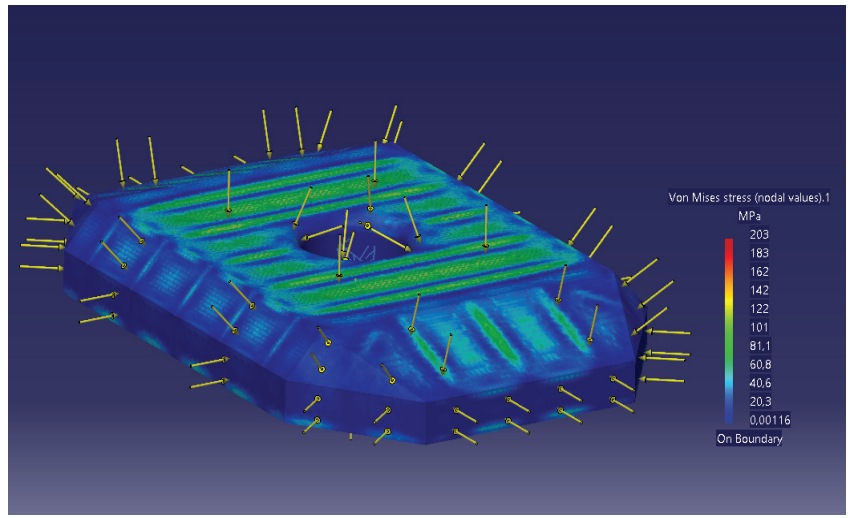

Figure 3.

Buoyancy block finite element method analysis using CAD software CATIA.

To achieve high thrust, precise positioning and movement in all directions for the five thrusters are used. The thrusters are DC brushless-motor-driven propeller type. The model used is Bluerobotics T200. Each thruster has the maximum power of $190 \mathrm{~W}$ at $12 \mathrm{VDC}$, and the current consumption of $25 \mathrm{~A}$ maximum. The maximum forward thrust force is $5.1 \mathrm{~kg}$, and the reverse thrust force is $4.1 \mathrm{~kg}$.

Two thrusters are used for the forward/backward thrust, two are for the lateral trust and one is used for the vertical thrust.

In this ROV design five Electronic Speed Controllers (ESC) are used to control the five thrusters, their speed and rotation. The thrust force is proportional to the average of the control voltage of the sub-sea microcomputer Pulse Width Modulated

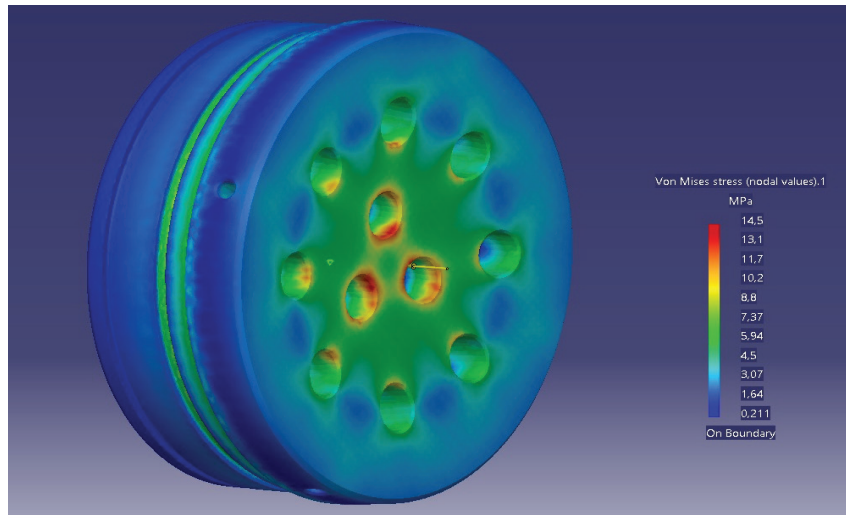

Figure 4.

Simulation of the pressure force applied to the vessel lid.

(PWM) output applied to the ESC. The ESC deals with the current up to $25 \mathrm{~A}$ and of 8-14 V voltage range. The five ESCs are located in the first pressure vessel.

To control thruster speed and rotation direction, the microcomputer program sets a width to the PWM pulses in the range from 1,100 to $1,900 \mu \mathrm{s}$. The pulse width of $1,500 \mu \mathrm{s}$ represents neutral controller position and corresponds to zero thrust power. Lower and higher pulse width corresponds to the thrust power as represented in Figure 5. The rotation direction is also pulse-width dependent. If the pulse width is above the neutral point, the forward thrust is achieved. The pulse width of the 1,900 $\mu$ s will provide the maximal forward thrust. If the pulse width is below the neutral point, the rotation direction reverses 


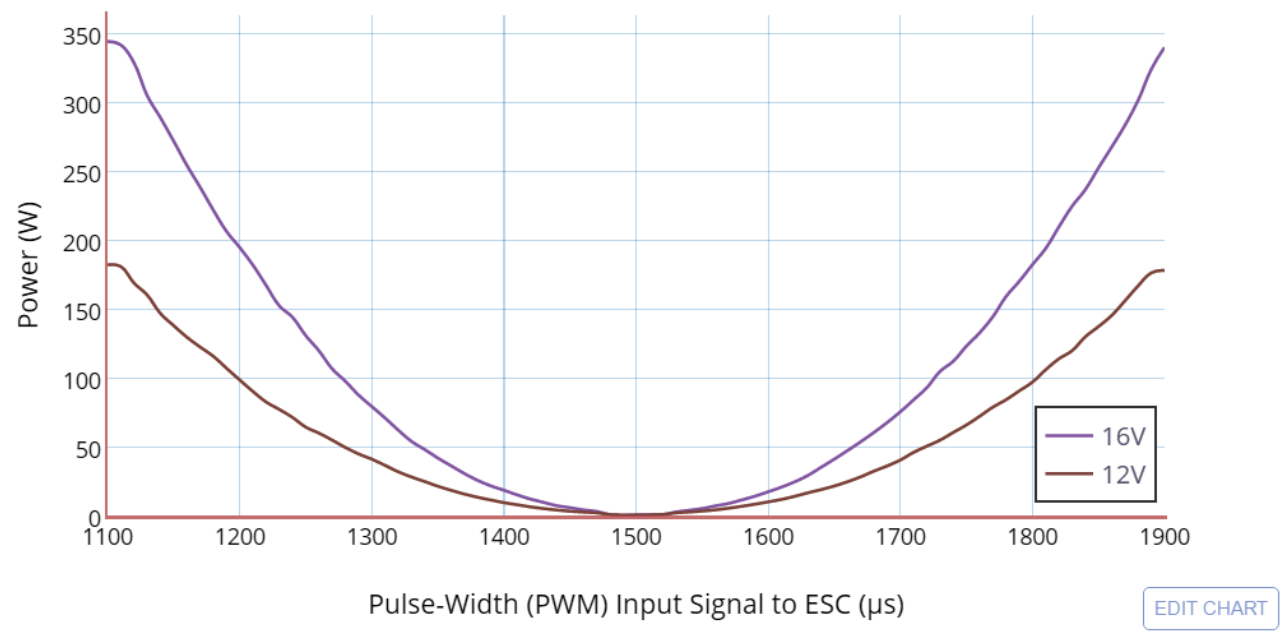

Figure 5 .

Thruster power vs. PWM input to ESC.

and the thrust is backward-oriented. The pulse width of $1,100 \mu \mathrm{s}$ will cause backward maximal thrust.

\section{ROV CONTROL AND VIDEO SYSTEM}

The control architecture (Figure 6) of the ROV outlined two main modules: the surface computer system and the sub-sea computer system. The computers used for the control system utilization are two Arduino class single board microcomputers (2549Q-AVR-02/2014 Datasheet) equipped with Ethernet shield and digital I/O shield (Figure 6). The aim of the microcomputers is to collect data from the control unit and sensors, and transfer it to the thrusters and the control unit respectively. The digital communication between the two microcomputers is realized using Ethernet network and Ethernet shield installed on board.

As the motion control interface, the PS2 compatible inexpensive joystick is selected to control the thruster power, thruster rotation speed, and rotation direction. Those parameters are determined by the position of the lever on the joystick and the appropriate buttons. The information from the joystick is transferred to the Arduino through PS2/USB interface.

Control knobs and levers on this joystick allow great versatility if additional functionalities are needed later on. The ROV's left and right rotational capability as well as its horizontal linear motion is determined by the position of the right and left levers respectively, while its vertical linear movement is determined by pressing the lever towards the base of the joystick. The joystick generates a series of numeric data corresponding to the positions of the stick and rotatable tab respectively. The data are further transferred from the surface microcomputer to the sub-sea microcomputer using Ethernet (Ferreira et al., 2014) and UDP protocol. UDP is simple "request-answer" protocol. The subsea Arduino sends request to the control Arduino. Upon receiving the request, the surface Arduino sends the answer that contains control data. If the data are not regularly received, the sub-sea computer continues to send requests. Similarly, the same process takes place in data transfer from the sub-sea microcomputer to the surface microcomputer.

There are two displays installed on the control box. The first one is a small LCD alphanumeric color display (Figure 8) used for the visualization of the real time sensor data. The second display is a standard $15^{\prime \prime}$ color display used for displaying video signal acquired by the sub-sea camera. The video signal transmission is realized by straightforward digital video streaming from the subsea camera to the display installed on the control box.

The ROV is equipped with five sensors. The sensors are connected to the Arduino analog inputs. The parameters of interest to be measured are depth, temperature and compass direction. To detect a leak in vessels, each electronic vessel has a pressure sensor which is also connected to the Arduino analog input. 


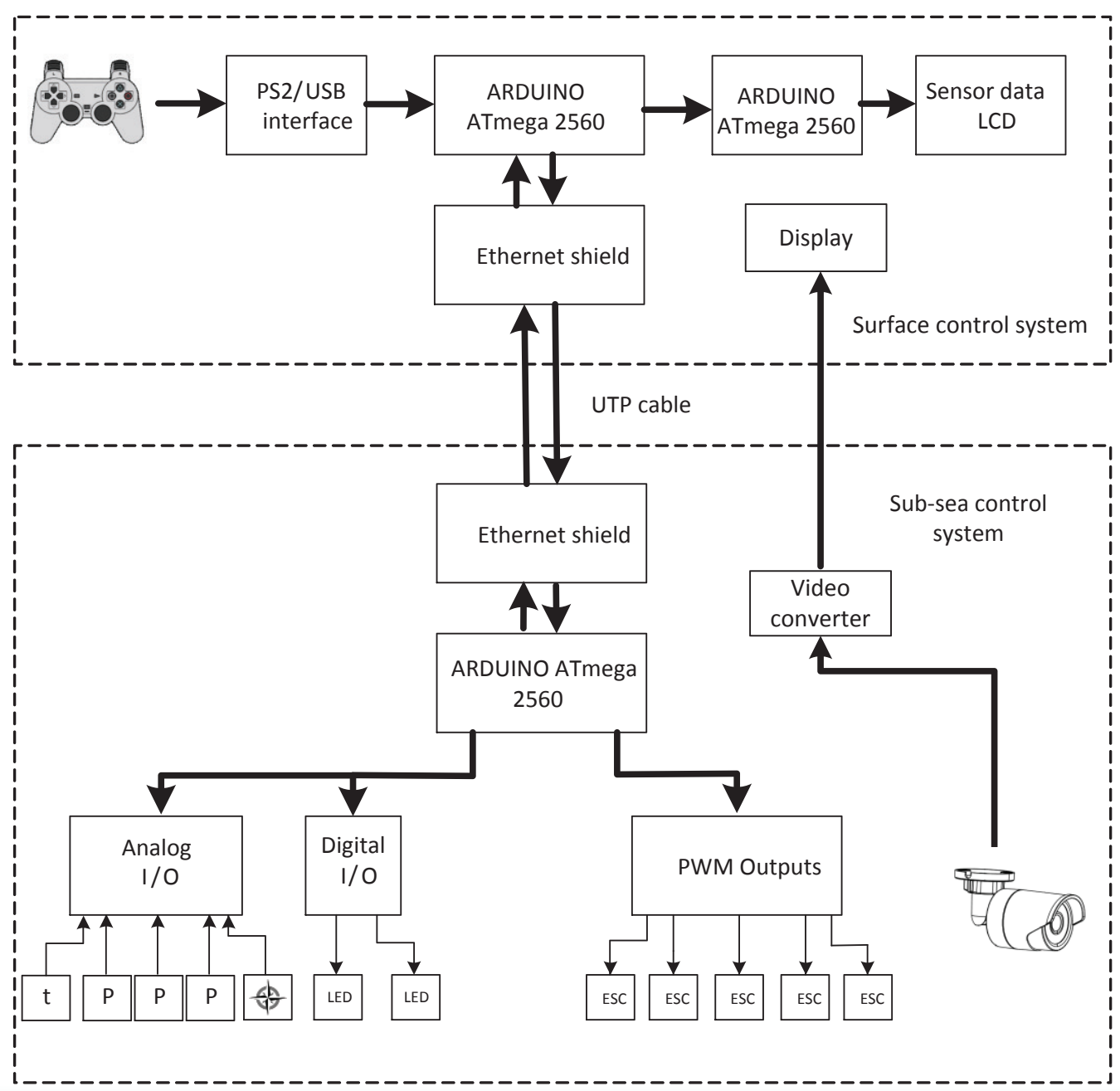

Figure 6.

ROV control and video system layout scheme.

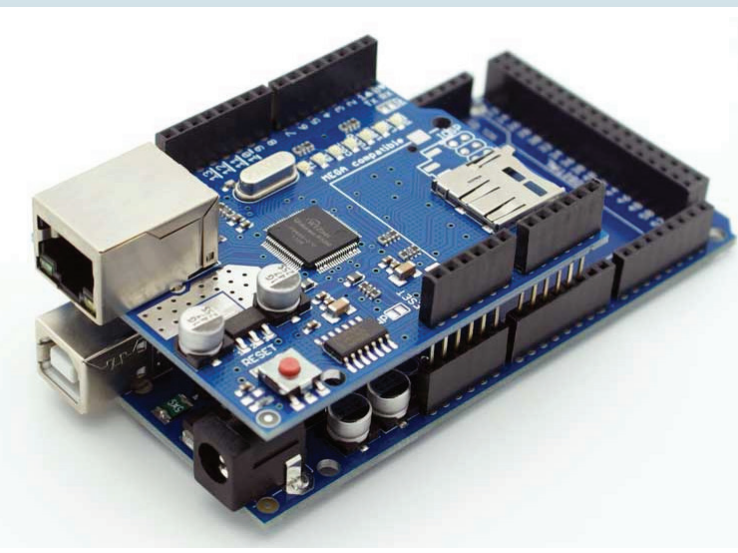

Figure 7.

Arduino ATmega single board computer.

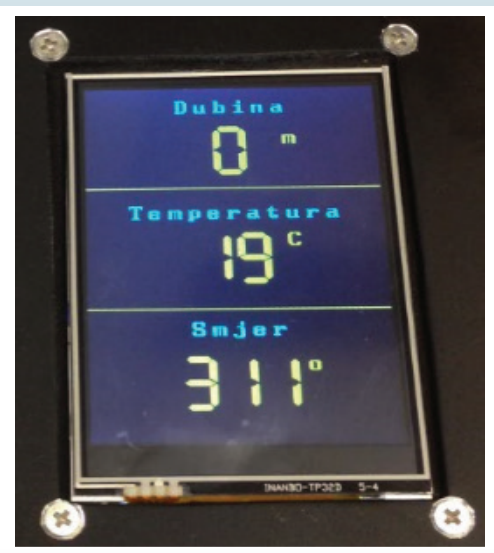

Figure 8.

Key parameters data LCD. 


\section{THE ROV'S POWER SUPPLY SYSTEM}

The ROV's power system is designed to deliver DC voltage to the control and sub-sea microcomputers, sensors, display, camera and five thrusters from 220V AC mains voltage (Figure 9). The power required by the electronic systems is moderate, and it is approximately $15 \mathrm{~W}$. On the contrary, the power required by the five thrusters is significant. It is considered the worst case when four thrusters simultaneously operate at full power. In that case, the required power is little less than $1,000 \mathrm{~W}$. In order to minimize the copper losses and copper cable cross section, the 100V DC link voltage is selected. Since the ESCs deal with voltage range of 8-14 V DC, each ESC and thruster are powered by dedicated 100/12 V DC/DC switch mode converter. The sub-sea electronics is powered by an additional sixth 100/12 V DC converter. All six converters are located into the second vessel. To obtain the desired power, the two $500 \mathrm{~W}$ switch mode AC/DC converters are connected in parallel and installed in the control box. An additional AC/DC converter with $12 \mathrm{~V}$ and $5 \mathrm{~V}$ outputs is used for powering the electronics. The display has its own embedded converter and is directly connected to the mains.

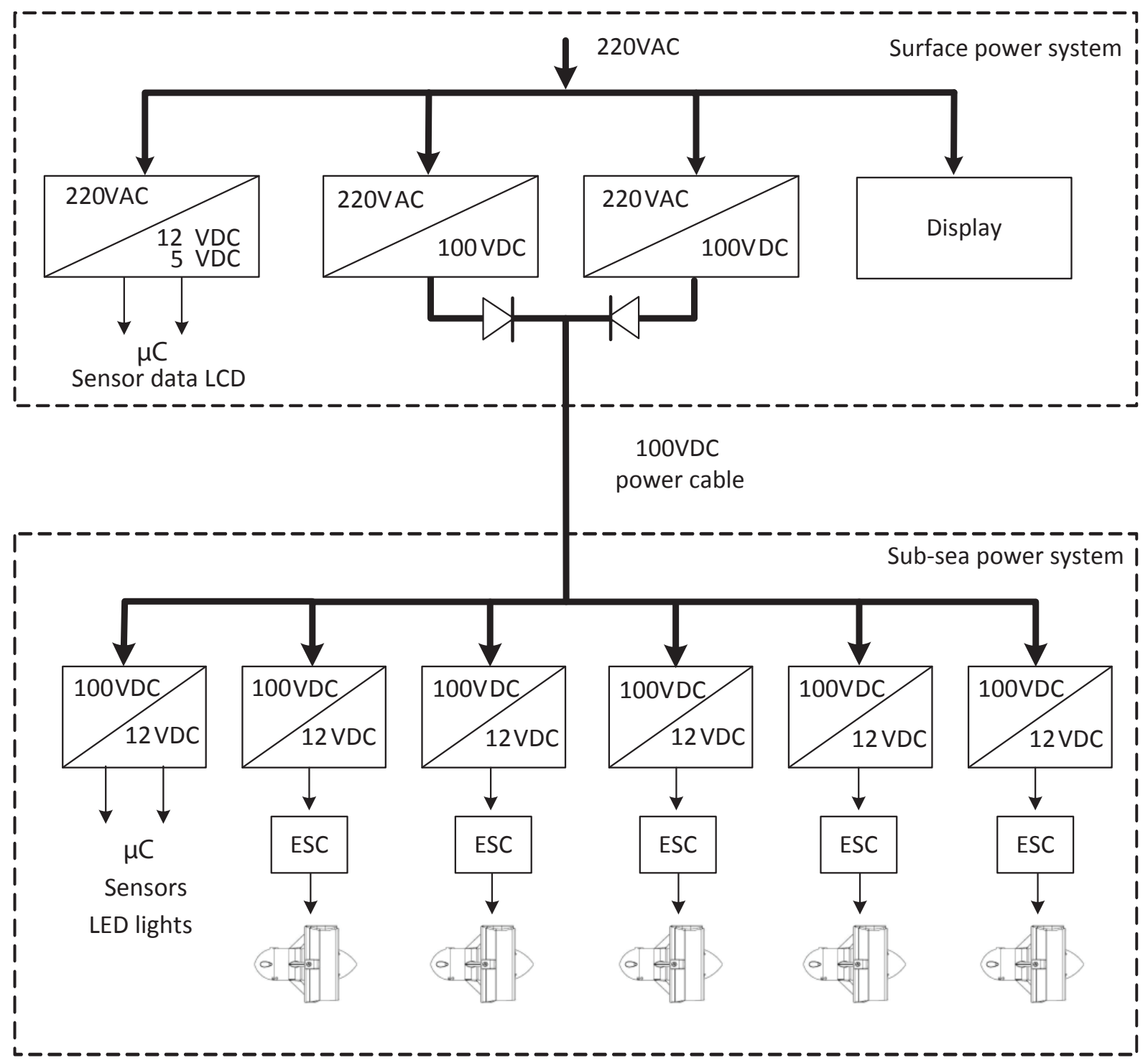

Figure 9

Power system layout scheme. 


\section{DESIGN AND IMPLEMENTATION}

Drawings and parameter calculation on the steel frame and aluminum buoyancy block were made in house, by the students in the Mechanical Department laboratory. Electronic components, microcomputer, ESCs, thrusters are off-the-shelf components bought on the market. The electrical project and software were written and tested by the Electrical Engineering department students.

The umbilical cable is composed of three different cables. Power cable, UTP cable, and video cable are merged together by plastic clips. The small, support, polyurethane blocks are installed along the cable set to achieve weight compensation and cable buoyancy.

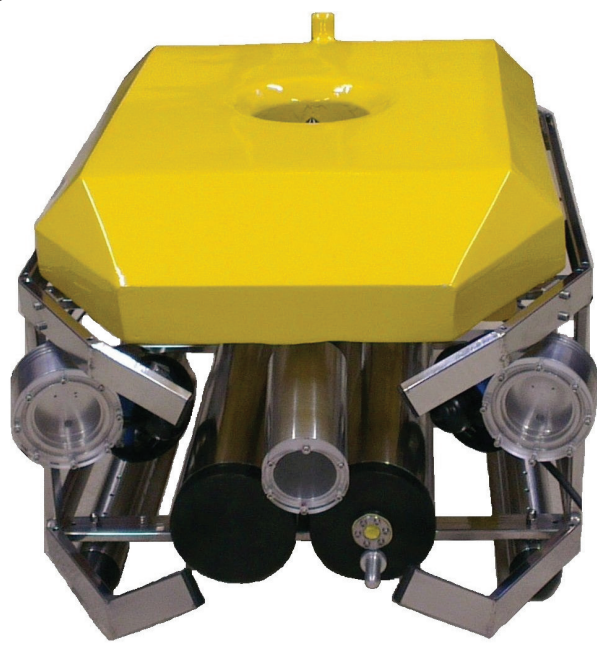

Figure 10

ROV frame, buoyancy block and vessels.

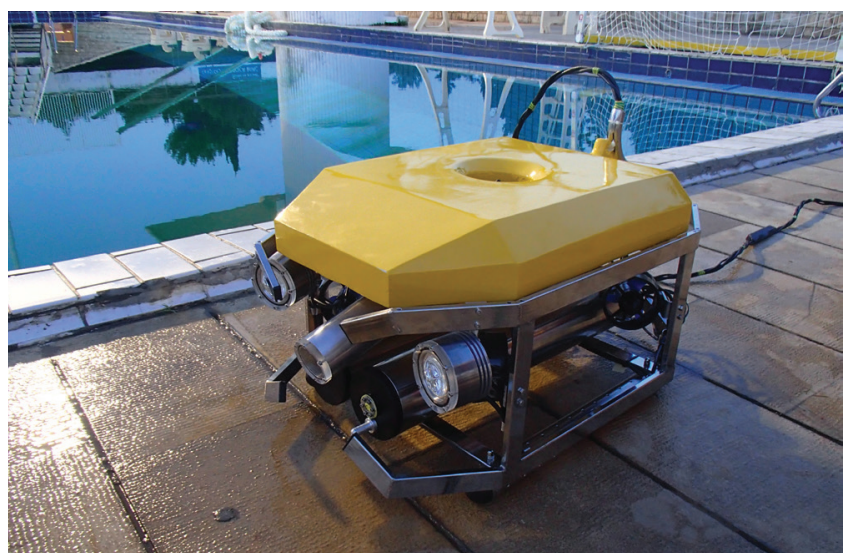

Figure 11.

ROV during wet testing at the University swimming pool.
The ROV has been wet-tested in the University swimming pool. After that, the ROV passed a few tests in the $150 \mathrm{~m}$-deep sea. The ROV's size is $0.65 \times 0.45 \times 0.40 \mathrm{~m}$, whilst the total mass, without the cable, is $15 \mathrm{~kg}$.

\section{CONCLUSION}

In this project, the low cost Commercial off-the-shelf (COTS) components and in-house development were used to utilize the ROV capable of diving down to the depth of up to $150 \mathrm{~m}$. The ROV shows excellent stability and maneuverability. The control software is also in-house-developed by students to meet the hardware needs.

It is a robust and modular underwater ROV with wide functionality. The ROV can be easily customized and deployed in a relatively short period of time.

The future work will be focused on the implementation of the robot manipulator arm and redesign of the umbilical cable. By using high-frequency power line communication system, a single pair of wires can be used to transfer both data and power at the same time. This will simplify the design and further reduce the cost and complexity of the umbilical cable. Such communication systems are under consideration.

The realized ROV platform will be used by the company PLOVPUT LLC. for the sub-sea power electrical cable inspection, and by UNIST as a demonstration of the advanced electrical and control systems.

\section{REFERENCES}

ATmega640/V-1280/V-1281/V-2560/V-2561/V, (2014), Datasheet 2549Q-AVR02/2014, Atmel corporation.

Dobrilo L., (2011), Strukturna analiza ronilice od kompozitnih materijala primjenom metode konačnih elemenata, BSc Thesis, Zagreb: University of Zagreb, Faculty of Machine Engineering and Naval Architecture.

Yusoff, M. A. M. and Arshad, M. R. A., (2009), Development of Small Class Remotely Operated Vehicle for Underwater Monitoring and Inspection, The 2nd International Conference of Underwater System Technology (USYS'8), Bali, November 2, available at: http://urrg.eng.usm.my/ppr/2009/Development_of_Small_Class_Remotely_ Operated_Vehicle_For_Underwater_Monitoring_and_Inspection.pdf

Christ R. D. and Wernli R. L., sr., (2014), A User Guide for Remotely Operated Vehicles, Elsevier Ltd.

Christ R. D. and Wernli R. L., sr., (2007), The ROV Manual: A User Guide for Observation Class Remotely Operated Vehicles, Elsevier Ltd.

Unknown, ROV history, available at: http://www.rov.org/rov_history.cfm, [accessed 14 January 2017.].

More W. S., Bohom H., Jensen V., (2010), Underwater Robotics: Science, Design \& Fabrication, Monterey, CA: Marine Advanced Technology Education (MATE) Centre.

Ferreira C. Z., Conte G. Y. C., Avila J. P. J., Pereira R.C.., Ribeiro T. M. C., (2014), Underwater robotic vehicle for ship hull inspection, ABCM Symposium Series in Mechatronics, 6, pp. 622 - 632., available at: http://www.abcm.org.br/upload/files/ PI_V_02\%281\%29.pdf 